CORRECTION

https://doi.org/10.1038/s41586-018-0271-3

\title{
Publisher Correction: ANKRD16 prevents neuron loss caused by an editing-defective tRNA synthetase
}

My-Nuong Vo, Markus Terrey, Jeong Woong Lee, Bappaditya Roy, James J. Moresco, Litao Sun, Hongjun Fu, Qi Liu, Thomas G. Weber, John R. Yates III, Kurt Fredrick, Paul Schimmel \& Susan L. Ackerman

Correction to: Nature https://doi.org/10.1038/s41586-018-0137-8, published online 16 May 2018.

In this Article, errors were inadvertently introduced in Figs. 3 and 4 during the production process. In the Fig. $3 \mathrm{~b}$ western blot, 'MycAlaRS' in row one should have been 'Myc-AAD Aars', 'AlaRS' in row two should have been 'Aars', and 'ANKRD16' in row four should have been 'Ankrd16. In Fig. 4f, the labels 'ANKRD16' and 'ANKRD16(3xR)' should have been 'Ankrd16' and 'Ankrd16 ${ }^{3 \times R}$ ', respectively. In addition, in the Fig. $3 c$ western blot, the position of the molecular mass markers had shifted. These figures have been corrected online, and the original, incorrect figures are shown as Supplementary Information to this Amendment for transparency.

Supplementary Information is available for this Amendment at https://doi.org/ 10.1038/s41586-018-0271-3 